\title{
Performance Evaluation for Medical Images Using Partitioned Iterated Function Systems
}

\author{
Pradeep kumar.B.P. ${ }^{1}$ Prathap $\mathrm{C}^{2}$ \\ Department of Electronics \& Communication Engineering Akshaya Institute of Technology, Tumkur, \\ Karnataka, India
}

\begin{abstract}
The compression of medical images is essential for reducing the cost of data storage and transmission time which in turn helps in better utilization of Bandwidth. The demand for images, video sequences and computer animation has increased drastically over the years which have also resulted in image and video compression. Image compression is broadly classified into lossy and lossless compression. Fractal image compression (FIC) is a lossy compression method. In fractal image compression an image is coded as a set of contractive transformations in a complete metric space. The set of contractive transformations is guaranteed to produce an approximation to the original image. In this paper quad-tree FIC is implemented on different Imaging modalities like Medical Resonance (MR) Image of Brain, Computerized tomography(CT) of Bone.The quality factors like Mean Square Error (MSE), Peak Signal-to-Noise-Ratio (PSNR) Compression ratio $(C R)$, Encoding time and decoding time for different imaging modalities with different threshold values are analyzed in this paper. From the matlab simulated results it is observed that Quad-tree FIC works better on medical image as it provides better PSNR, CR values over the other images. This paper also includes a comparison between standard FIC and Quad-tree FIC on MR image of Brain and study of the parameters reveals that Quad-tree FIC works better than Standard FIC.

Keywords - Medical Imaging Fractal image compression, Quad-tree partitioning, objective quality measures
\end{abstract}

\section{INTRODUCTION}

Fractal was first introduced in geometric field and the birth of fractal geometry was traced by IBM mathematician Benoit. B. Mandelbrot. Later M. Barnsley introduced this idea to data and images using iterated function systems (IFS) in 1988 [1] which was not practically us eful and later A.E. Jacquin modified these IFS into partitioned IFS. Fractal image compression is also called as fractal image encoding because compressed image is represented by contractive transforms and mathematical functions are required for reconstruction of original image. Contractive transforms ensures that, the distance between any two points on transformed image will be less then the distance of same points on the original image [1]. These transforms are composed of the union of a number of affine mappings on the entire image, known as iterated function system (IFS) [1], [2]. Barnsley derived a special form of the Contractive Mapping Transform (CMT) applied to IFS's called the College Theorem [3,4]. The usual approach of fractal image compression is based on the college theorem, which provides distance between the image to be encoded and the fixed point of a transform, in terms of the distance between the transformed image and the image itself. This distance is known as college error and it should be as small as possible. A.E. Jacquin gave first publication on Fractal image compression with partitioned IFS (PIFS) in 1990 [2],[3], [4]. In Jacquin's method the image is partitioned in sub images called as 'Range blocks' and PIFS are applied on sub-images, rather than the entire image. Locating the range blocks on their respective position in image itself forms the entire image. Temporary images used to form range blocks are known as domain blocks. As opposed to most of other compression methods, the fractal coding is asymmetric. From one hand, it is a drawback because encoding lasts much longer that in other methods. But at the same time it is an advantage because the decoding process is very fast - it takes usually less time to decode an image with fractal method than to read the same image, but uncompressed, from the hard drive. This feature is useful when the image must be sent through the Internet another feature of fractal compression that attracts one's attention is the greatness of compression ratios that can be achieved with this method. Since it is a lossy method, it gives much smaller compressed file than any lossless compression algorithm. However, the medical images cannot be compressed with too high compression ratio because the loss of informat ion can turn out to be too high.

In this paper the quad-tree fractal image compression [[5] has been applied on different imaging modalities like CT and MRI. The compression ratio(CR),Peak Signal to Noise ratio (PSNR),Mean Square Error(MSE),encoding time, decoding time are obtained for the images by varying the threshold of quad-tree partitioning and they are analyzed. 
This paper is organized as follows. Section 2 briefs about the standard fractal image compression method. Section 3 explains the proposed Fractal coding algorithm. Section 4 deals with Implementation and quality factors. Section 5 gives results, Section 6 derives Conclusion

\section{FRACTAL IMAGE COMPRESS ION}

Imagine a special type of photocopying machine that reduces the image to be copied by a half and reproduces it three times on the copy.

Figure 1 shows this. Now, feed the output of this machine back as input. Figure 2 shows several iterations of this process on several input images. We observe that all the copies seem to be converging to the same final image, the one in figure 2(c). We call this image the attractor for this copying machine. Because the copying machine reduces the input image, any initial image will be reduced to a point as we repeatedly run the machine. Thus, the initial image placed on the copying machine doesn't affect the final attractor; in fact, it is only the position and the orientation of the copies that determines what the final image will look like. Since it is the way the input image is transformed that determines the final result of running the copy machine in a feedback loop, we only describe these transformations to be contractive - that is, a given transformation applied to any two points in the input image, it must bring themclosertogether in the copy. In practice, choosing transformations of the form

$$
w_{i}\left[\begin{array}{l}
x \\
y
\end{array}\right]=\left[\begin{array}{ll}
a_{i} & b_{i} \\
c_{i} & d_{i}
\end{array}\right]\left[\begin{array}{l}
x \\
y
\end{array}\right]+\left[\begin{array}{l}
e_{i} \\
f_{i}
\end{array}\right]
$$

is sufficient to yield rich and interesting set of attractors. Such transformations are called affine transformations [5][6] of the plane, and each can skew, stretch, rotate, scale and translate an input image.

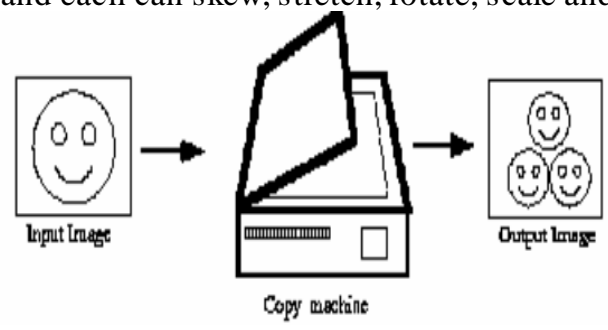

Fig. 1. A copy machine that makes three reduced copies of an input image

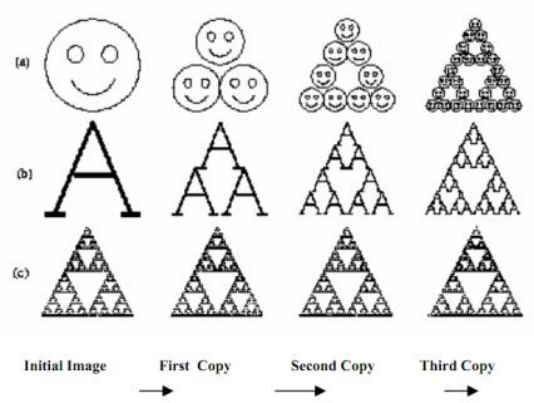

Fig. 2. The first three copies generated by the copying machine of fig. 1 .

The transformation produces modification of the pixel values inside the block. It allows changing the gray level information in order to get good approximation of the Range block $R_{i}$ In the implementation, we will consider only 8

possible shuffles of pixels $\left[a_{i}, b_{i}, c_{i}, d_{i}\right]$, a contrast scaling $s_{i}$ and brightness shift $o_{i}$. The transformation can therefore be expressed as

$$
\mathrm{Si}\left[\begin{array}{l}
x \\
y \\
z
\end{array}\right]=\left[\begin{array}{ccc}
a_{i} & b_{i} & 0 \\
c_{i} & d_{i} & 0 \\
0 & 0 & s_{i}
\end{array}\right]\left[\begin{array}{c}
x \\
y \\
z
\end{array}\right]+\left[\begin{array}{c}
0 \\
0 \\
o_{i}
\end{array}\right]
$$

\section{A.Fractal Encoding Algorithm}

- Load an input image into buffer;

- Partition the image into square blocks with non overlap (as Range blocks ); 
- Choose initially the size of the do main block to be twice the size of the range block;

- Down sample the domain blocks to the size of range blocks and compute the eight possible affine transformations for each block;

- Choose the domain block that resembles the range block with respect to some metric and compute the encoding parameters that satisfy the mapping;

- Save the coefficients which represents fractal element.

\section{A. Fractal decoding algorithm:}

- Load the initial image which is to be decoded;

- Apply $w_{i}$ repeatedly until we converge to a fixed point which means for each $w_{i}$ we find the domain block, rescale to the size of range block;

- Multiply the pixel values by scaling factor $S_{i}$ then add offset value $o_{i}$ and compute the pixel values in each $R_{i}$, which allows copying the content of the domain blocks to the range blocks;

- Take the output of first iteration (Range block) to be the input of the next iteration;

- $\quad$ Repeat doing the same until the desired attractor is reached.

One of the most notable features of fractal image compression is that the decoding process is simple. The decoder proceeds its work in the same way as in the case of the traditional encoder (i.e., fixed block size encoding). The decoder consumes less time for computation compared to that of an encoder. The decoding time generally depends on the number of Iterations and here it takes only few iterations ranging from $4-8$ to reach the fixed point.

\section{QUAD-TREE PARTITIONING}

The most popular partitioning mechanis $\mathrm{m}$ is obtained by partitioning the image in a tree structure. A quad-tree partitioning[6] is a representation of an image as a tree in which each node corresponding to a square portion of the image contains four sub-nodes corresponding to the four quadrants of the square, the root of the tree being the initial image as in figure 3 shown below.

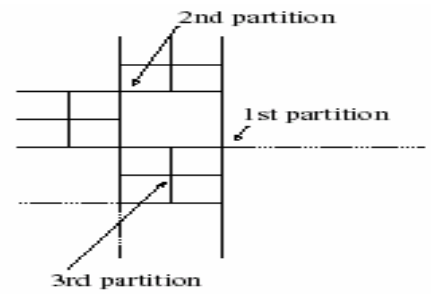

Fig. 3 Quad-tree Partitioning

\section{A. Algorithm:}

- Read The Input image to be compressed;

- Scale the Image and Divide the image ( in domain ) into non overlapping domain with the block size of 16x16.The Size of the domain is twice the range blocks.;

- Partition the orig inal image in range according to quad-tree method which divides a square image into four equal-sized square blocks, and then test each block to see if it meets some criterion of homogeneity. If a block meets the criterion, it is not divided any further. If it does not meet the criterion, it is subdivided again into four blocks, and the test criterion is applied to those blocks. This process is repeated iteratively until each block meets the criterion. The result may have blocks of several different sizes. The criteria met in the present quad-tree method is that the method will not produce blocks smaller than the minimum dimension of block, even if the resulting blocks do not meet the threshold ;

- Apply Affine transformation to each of the domain block and compare with each of the range block and find a closest approximation of the do main block with that of the range block;

- The transformed domain block which is found to be the best approximation for the current range block is assigned to that range block forms the fractal code book;

- The reconstruction process of the original image consists on the applications of the transformations describe in the fractal code book iteratively to some initial image until the encoded image is retrieved back. PSNR, CR[7], Encoding time Decoding time and MSE is calculated for the further analysis of the image. 


\section{IMPLEMENTATION AND QUALITY ASS ESS MENT}

\section{A. Implementation}

Forty images out of which twenty images of Magnetic Resonance Images[8] of brain(T1 and T2 weighted) and twenty images of CT images [8][9] of bones of size 256x256 of 8 bit gray scale where collected from JSS Hospital and Vikram Hospital Mysore. Fractal image compression using quad-tree partitioning was applied on the images with the threshold varying between 0.1 and 0.4 . The Minimum dimension of the block was taken to be 2 and 4. The compression Ratio, PSNR, MSE, Encoding and decoding time were computed for the images. The standard FIC and Quad-tree FIC was applied on the MR image of Brain and a comparison of the PSNR, encoding time and decoding time was made.

\section{B. Quality Assessment}

The image quality can be evaluated objectively and subjectively. Subjective measures are based on observer's response such as Mean Opinion Score (MOS) and objective measures are based on computable distortion measures such as reconstruction error, Mean Square Error (MSE) and PSNR. Among the objective numerical measures of picture quality that are based on computable distortion measures like mean square error, peak signal to noise ratio and Compression Ratio are considered in this work.

PSNR: The PSNR [9] computes the peak signal-to-noise ratio, in decibels, between two images. This ratio is often used as a quality measure ment between the original and a compressed image. The higher the PSNR, the better the quality of the compressed or reconstructed image. The Mean Square Error (MSE) and the Peak Signal to Noise Ratio (PSNR) are the two error metrics used to compare image compression quality. The MSE represents the cumulative squared error between the compressed and the original image, whereas PSNR represents a measure of the peak error. The lower the value of MSE, the lower is the error.

The MSE and PSNR are calcu lated using the following equations;

$$
\begin{aligned}
& M S E=\frac{1}{M N} \sum_{I=0}^{M-1} \sum_{J=0}^{N-1}\left(f^{\prime}(i, j)-f(i, j)\right]^{2} \\
& P S N R=10 \log \left[\frac{255^{*} 255}{M S E}\right] d B
\end{aligned}
$$

In the previous equation, $M$ and $N$ are the number of rows and columns in the input images, respectively. $\mathrm{f}(\mathrm{i}, \mathrm{j})$ is the input image and $f^{\prime}(\mathrm{i}, \mathrm{j})$ is the reconstructed image.

\section{V RESULTS AND CONCLUSIONS}

\section{A. Simulated Results}

The above algorithms are programmed using MATLAB 7.12 and the programs are executed on PC computer with CPU speed equal to 2.2 GHZ, core2duo,2GB RAM.

The performance of compression in terms of PSNR, for MR images of Brain. for different thresholds and minimu $\mathrm{m}$ dimension of 2 is shown in Table 1

\begin{tabular}{|c|c|c|c|c|c|}
\hline Imag & \multicolumn{5}{|c|}{ PSNR For different thresholds in dB } \\
\hline \multirow{5}{*}{$\begin{array}{l}\text { MRI } \\
\text { of } \\
\text { brain }\end{array}$} & $\begin{array}{l}\text { Sl. } \\
\text { no }\end{array}$ & $\begin{array}{l}\text { Th=0 } \\
.1\end{array}$ & $\begin{array}{l}\text { Th }=0 \\
.2\end{array}$ & $\begin{array}{l}\text { Th }=0 \\
.3\end{array}$ & Th $=0.4$ \\
\hline & 1 & 32.9 & 32.7 & 31.8 & 31.0 \\
\hline & 2 & 32.6 & 32.4 & 31.6 & 30.8 \\
\hline & 3 & 32.8 & 32.6 & 31.9 & 30.9 \\
\hline & 4 & 32.6 & 32.4 & 31.6 & 30.9 \\
\hline \multicolumn{2}{|c|}{ average } & 32.7 & 32.5 & 31,7 & 30.9 \\
\hline \multirow{4}{*}{$\begin{array}{l}\text { CT of } \\
\text { bone }\end{array}$} & 1 & 32.1 & 31.9 & 31.6 & 31.2 \\
\hline & 2 & 34.6 & 34.4 & 33.7 & 33.4 \\
\hline & 3 & 32.8 & 32.7 & 32.4 & 32.2 \\
\hline & 4 & 36.4 & 36.2 & 35.8 & 35.1 \\
\hline \multicolumn{2}{|c|}{ Average } & 33.9 & 33.8 & 33.3 & 32.9 \\
\hline
\end{tabular}

\section{TABLE.1: PERFORMANCE OF PSNR FOR MEDICAL IMAGES}

Threshold=0.1( $\mathrm{T} 1$ weighted image MR Image Of Brain) 


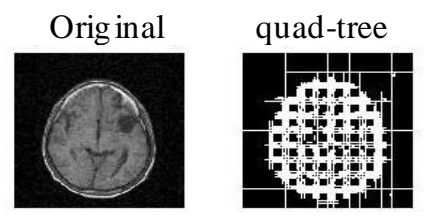

output Image

Threshold $=0.1(\mathrm{CT}$ of Bone $)$
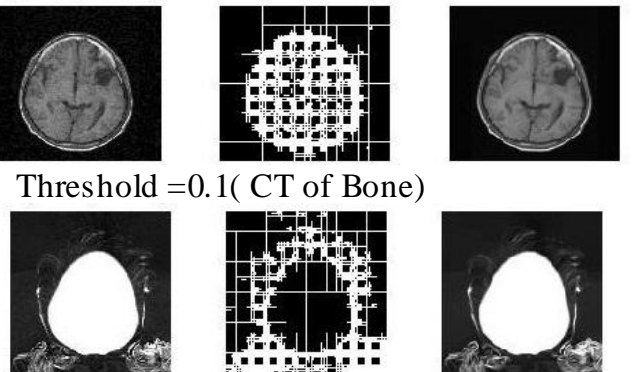

Threshold $=0.4(\mathrm{CT}$ of Bone $)$
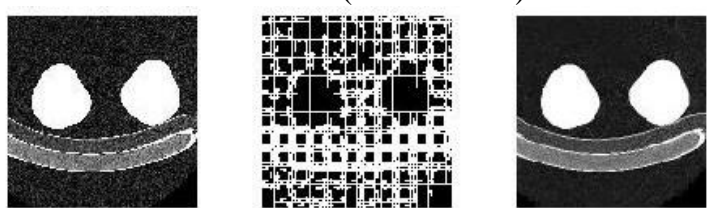

Fig.4: Results of quad-tree FIC applied on MR Image and CT

The Figure 4 are the results obtained by applying quad-tree FIC on MR Image of Brain and CT Of Bone for threshold of 0.1 and 0.4 .The MR Image is aT1 weighted normal image .

The Performance of compression in terms of CR, for MR images of Brain and CT OF bone.for different thresholds and minimumdimension of 2 is shown in Table 2

TABLE .2: PERFORMANCE OF CR FOR MEDICAL IMAGES

\begin{tabular}{|l|l|l|l|l|l|}
\hline Images & \multicolumn{5}{l}{ CR For diffe rent th resholds } \\
\hline \multirow{2}{*}{$\begin{array}{l}\text { MRI } \\
\text { OF } \\
\text { brain }\end{array}$} & Sl:no & Th=0.1 & Th=0.2 & Th=0.3 & Th=0.4 \\
\cline { 2 - 6 } & 1 & 5.3 & 6.5 & 7.7 & 8.2 \\
\cline { 2 - 6 } & 2 & 5.1 & 6.2 & 7.4 & 8.0 \\
\cline { 2 - 6 } & 3 & 5.0 & 6.0 & 7.2 & 7.9 \\
\cline { 2 - 6 } & 4 & 5.1 & 6.0 & 7.2 & 7.7 \\
\hline Average & 5.1 & 6.1 & 7.3 & 7.95 \\
\hline $\begin{array}{l}\text { CT of } \\
\text { bone }\end{array}$ & 1 & 5.4 & 6.5 & 7.0 & 7.4 \\
\cline { 2 - 6 } & 2 & 6.9 & 7.6 & 8.2 & 8.3 \\
\cline { 2 - 6 } & 3 & 6.7 & 6.9 & 7.2 & 7.4 \\
\cline { 2 - 6 } & 4 & 7.9 & 8.3 & 8.6 & 8.8 \\
\hline Average & 6.7 & 7.3 & 7.7 & 7.9 \\
\hline
\end{tabular}

The performance of compression in terms of MSE, for MR images of Brain. for different thresholds and minimu $m$ dimension of 2 is shown in Table. 3 .

TABLE .3: PERFORMANCE OF MSE FOR MEDICAL IMAGES

\begin{tabular}{|c|c|c|c|c|c|}
\hline \multirow{2}{*}{\begin{tabular}{|l|}
\multicolumn{2}{|l|}{ Images } \\
MRI
\end{tabular}} & \multicolumn{5}{|c|}{ MSE For different thresholds } \\
\hline & SI.no & $\begin{array}{l}\text { Th=0. } \\
1\end{array}$ & $\begin{array}{l}\text { Th=0. } \\
2\end{array}$ & $\begin{array}{l}\text { Th=0. } \\
3\end{array}$ & $\begin{array}{l}\text { Th=0. } \\
4\end{array}$ \\
\hline \multirow{4}{*}{$\begin{array}{l}\text { of } \\
\text { brai } \\
\text { n }\end{array}$} & 1 & 132.9 & 141.7 & 172.3 & 209.9 \\
\hline & 2 & 143.7 & 151.9 & 179.8 & 217.9 \\
\hline & 3 & 136.6 & 143.8 & $\overline{171.2}$ & 213.3 \\
\hline & 4 & 145.1 & 151.4 & 180.4 & 212.2 \\
\hline \multicolumn{2}{|c|}{ Average } & 139.5 & 147.2 & 175.9 & 213.2 \\
\hline \multirow{4}{*}{$\begin{array}{l}\text { CT } \\
\text { of } \\
\text { bone }\end{array}$} & 1 & 162.4 & 169.8 & 190.3 & 197.8 \\
\hline & 2 & 90.4 & 95.1 & 110.8 & 120.6 \\
\hline & 3 & 137.6 & 139.8 & 145.1 & 158.6 \\
\hline & 4 & 59.7 & 62.7 & 69.6 & 80.4 \\
\hline \multicolumn{2}{|c|}{ Average } & 112.5 & 115.8 & 128.9 & 139.5 \\
\hline
\end{tabular}


The comparis on of performance of compression in terms of encoding time and decoding time between the standard FIC and quad-tree FIC for the MR images of Brain is show in Table 4

TABLE .4:COMPARSION OF ST ANDARD FIC AND QUAD-TREE FIC MR IMAGE OF BRAIN.

\begin{tabular}{|l|l|l|l|l|l|l|l|l|}
\hline $\begin{array}{l}\text { Test } \\
\text { Ima } \\
\text { ge }\end{array}$ & \multicolumn{3}{|l|}{ Standard FIC } & \multicolumn{4}{|l|}{ Quad-Tree FIC } \\
\hline & PSN & C & E & D & PS & C & E & D \\
& R & R & T & T & NR & R & T & T \\
\hline MRI & 23.6 & 94. & 10 & 3. & 30. & 6. & 7. & 6. \\
& & 8 & 9 & 7 & 6 & 61 & 5 & 8 \\
\hline
\end{tabular}

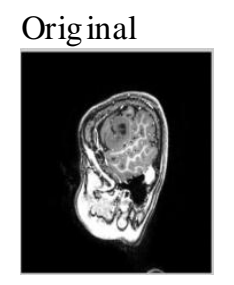

Standard FIC Decoded
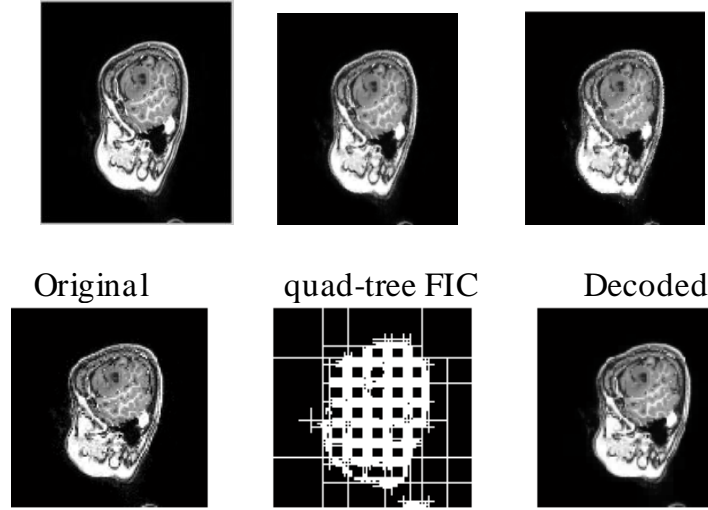

Decoded

Fig.5: comparis ons of standard FIC and Quad-tree FIC

The Figure 5 are the results obtained after applying Standard FIC and quad-tree FIC on MR images and its performance comparis on is being shown in table 4 .
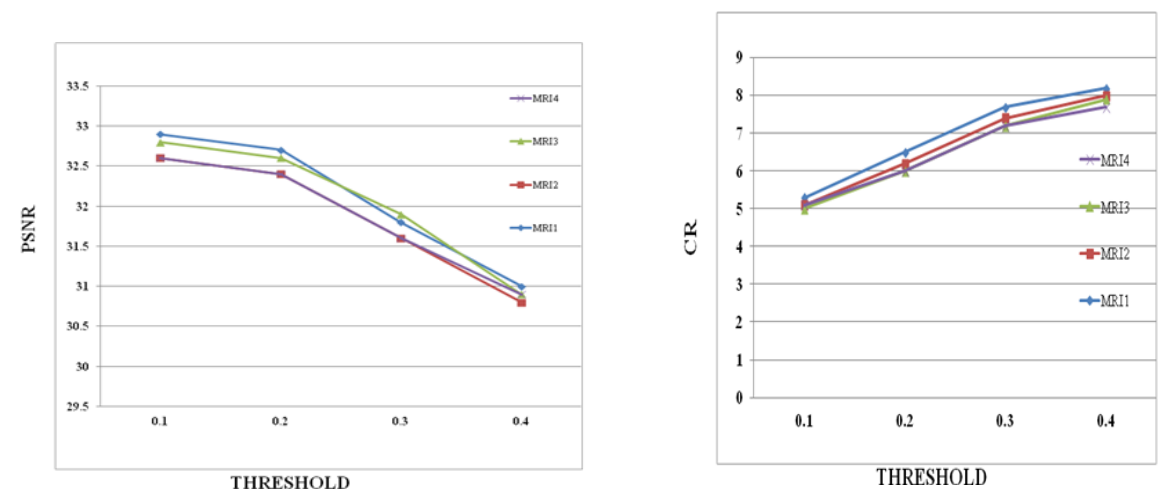

Fig :6. Variation of PSNR and CR with respect to threshold for MR Images

Figure 6 is a graph plotted from the results so obtained after applying quad-tree FIC on four T1 weighted MR Images of Brain and four CT Images of Bone. From the Graph it is observed that as the PSNR values decreases as the threshold value increases and as the Threshold value increases the CR increases. Also, the PSNR and CR are inversely proportional to each other.

\section{CONCLUS IONS}

- $\quad$ The PSNR Decreases with increase in threshold values for MR Images and CT images. The PSNR and threshold are inversely proportional.At low Threshold the PSNR is high. As the PSNR decreases with increase in threshold, the quality of the images shall not be good at high threshold. Hence an optimum threshold should be chosen without hampering the quality of the image.

- $\quad$ The PSNR value is high for quad-FIC method than standard FIC value for MR Image of Brain. Hence the quality of the image is better when quad-tree FIC is applied.

- The Compression Ratio increases with increase in threshold value. The CR and threshold are directly proportional. The compression Ratio is high for high threshold. High Compression Ratio is not advise able for Medical Images as much of the information shall be lost. 
- The average encoding time and decoding time for MR images using quad-tree FIC are $6.7 \mathrm{sec}$ and $5.8 \mathrm{sec}$ while the encoding time and decoding time for MR images using standard FIC are $109 \mathrm{sec}$ and $3.7 \mathrm{sec}$ respectively.

- $\quad$ The Mean square error increases with increase in threshold.

- The Quad-tree FIC works better than standard FIC for medical images as the encoding time and compression Ratio is less which is very important for of medical images The CR is high for CT images than MR image of Brain.

- $\quad$ As The CR increases the PSNR decreases.

\section{REFERENCE}

[1] M. Barnsley, Fractals Everywhere. New York: Academic,(1988).

[2] A.E. Jacquin, "Image coding based on a fractal theory of iterated contractive image transformation", IEEE Trans. On Image Processing, 1(1): (1992

[3] Y. Fisher, Fractal Image Compression: Theory and Application. New York: Springer-Verlag, (1994).

[4] A.E Jacquin, "Fractal image coding: A review", Proceeding of tile IEEE, 81(10): (1993)

[5] M.S.Soyjaudah and I.Jahmeerbacus "Fractal image compression using quad-tree partitioning" International Journal of Electrical Engineering Education 39/1

[6] Dr. Fakhiraldeen H. Ali Quad-tree Fractal Image Compression University of Mosul

[7] Sumathi Poobaland G. Ravindran, "Arriving at an OptimumValue of Tolerance Factor for Compressing Medical Images," wo rld Academy of Science,Engineering and Technology, vol. 24, pp. 169-173, 2006.

[8] Pamela Cosman, Gray R.M. and Olshen A.(1994b)“Evaluating Quality of Compressed Medical Images: SNR, Subjective Rating and Diagnost ic Accuracy', Proc. of the IEEE, Vol. 82, pp. 920-931.

[9] S. Bhavani et. al. / (IJCSE) International Journal on Computer Science and Engineering Vol. 02, No. 05, 2010, 1429-1434 A Survey On Coding Algorithms In Medical Image compression 